\title{
GENERALIZED RAY THEORY FOR DIPPING STRUCTURE
}

\author{
By Tai-Lin Hong and Donald V. Helmberger
}

\begin{abstract}
In this study we relax the strong limiting condition of parallel layering which is usually assumed in seismic modeling by allowing dipping boundaries. We start with the derivation of generalized ray theory in a wedge-shaped medium with free and rigid boundaries. Then, through the development of the method of equivalent models and de-Hoop contours, we extend the theory to dipping structure with elastic boundaries. The effect of a dipping inferface over a half-space for the case of a line source is shown by a series of numerical models which include various angles of dip and source-to-receiver distances. Results for a line source situated below the layer indicate that, when the layer thickens toward the receiver, one obtains a wave form similar to the case where the source is actually in the layer. These features are produced by the combination of forward and backward traveling rays which can have super-critical reflections.
\end{abstract}

\section{INTRODUCTION}

The seismologist can find it difficult to apply elasticity theory to seismic-wave problems for many reasons. One difficulty often encountered is that physical constitution parameters have depth dependencies. This problem has been handled in many ways, one of which is by approximating the earth structure by a stack of homogeneous layers and applying the concepts of generalized ray theory. Although horizontally layered models have proven useful in many situations, one is constantly confronted with non-horizontal structures where the usual analytical methods are not applicable. Rather than rely on relatively expensive numerical techniques to study these types of problems, it would be useful to develop analytical approximations which can provide some insight along with the solution. We will discuss one such approximate technique for the treatment of wave motion in locally dipping structure in this study.

We introduce the method by considering an $S H$ line source in a wedge-shaped medium with a free surface and a rigid lower boundary. After applying the classical ray expansion, as discussed by Hudson (1963), we solve for the motion by application of the Cagniard-de Hoop technique. A localized coordinate system is adopted which conserves the de Hoop contour and can be easily modified to include elastic boundaries. We are therefore able to recover not only optical results but head waves and tunneling effects as well Unfortunately, we cannot determine the accuracy of this procedure sine there is no other analytical work available to do an independent check, but a comparison of our results with those of finite element methods is, in general, good.

\section{Generalized Ray Theory for the Wedge Problem}

For certain boundary conditions the rigorous solution for $S H$-wave propagation can be derived. Hudson (1963) solved the problem with one boundary free and the other boundary rigid. In this section we solve the same problems by the Cagniard-de Hoop method to obtain the solution in terms of generalized rays.

We assume a line source situated in a wedge as diagrammed in Figure 1 . The equa- 
tion of motion in terms of displacement for a whole space containing this source is simply

$$
\nabla^{2} \bar{W}_{0}-\frac{s^{2}}{\beta^{2}} \bar{W}_{0}=\frac{\delta(R)}{R} \cdot \frac{-\bar{f}(s)}{2 \pi}
$$

where "-" is the Laplace transform function, $s$ is the Laplace variable $W_{0}$ is the $S H$ displacement, and $f(t)$ is the source time function.

By setting $\bar{f}(s)=2 \pi$, we have

$$
\bar{W}_{0}=K_{0}(\rho R)
$$

where $\rho=s / \beta$, and $K_{0}$ is the modified Bessel function of order zero.

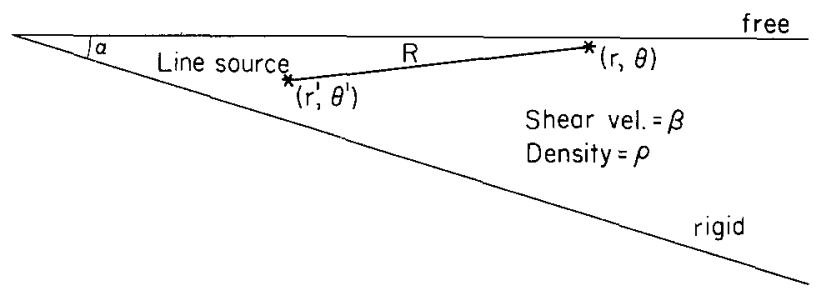

FIG. 1. Diagram of the problem setup with $\theta$ measured clockwise from the free surface. The distances from the tip of the wedge to the source and receiver are $r^{\prime}$ and $r$ with the separation given as $R$.

Following the transformation by Oberhettinger (1954), we obtain

$$
\bar{W}_{0}=-i \int_{-\infty}^{\infty} I_{-i \lambda}\left(\rho r^{\prime}\right) \cdot K_{i \lambda}(\rho r) \cdot \frac{\cosh \left[\lambda\left(\pi-\left|\theta-\theta^{\prime}\right|\right)\right]}{\sinh \pi \lambda} d \lambda .
$$

The homogeneous solution is assumed to have the following form

$$
\bar{W}_{r}=-i \int_{-\infty}^{\infty} I_{-i \lambda}\left(\rho r^{\prime}\right) \cdot K_{i \lambda}(\rho r) \cdot\left[f_{1}(\lambda) e^{\lambda \theta}+f_{2}(\lambda) e^{-\lambda \theta}\right] d \lambda
$$

The functions $f_{1}(\lambda)$ and $f_{2}(\lambda)$ are to be determined by the following boundary conditions

$$
\frac{\partial \bar{W}}{\delta \theta}=0, \text { at } \quad \theta=0
$$

and

$$
\bar{W}=0, \text { at } \theta=\alpha
$$

where, $\bar{W}=\bar{W}_{0}+\bar{W}_{r}$.

We obtain,

$$
\begin{aligned}
& f_{1}=\frac{-\sinh \left[\lambda\left(\pi-\theta^{\prime}\right)\right] e^{-\lambda \alpha}-\cosh \left[\lambda\left(\pi-\alpha+\theta^{\prime}\right)\right]}{2 \cosh (\lambda \alpha) \sinh (\lambda \pi)} \\
& f_{2}=\frac{-\cosh \left[\lambda\left(\pi-\alpha+\theta^{\prime}\right)\right]+\sinh \left[\lambda\left(\pi-\theta^{\prime}\right)\right] e^{\lambda \alpha}}{2 \cosh (\lambda \alpha) \sinh (\lambda \pi)} .
\end{aligned}
$$


Thus at a receiver with $\theta=0$, we have

$$
\bar{W}=-i \int_{-\infty}^{\infty} I_{-i \lambda}\left(\rho r^{\prime}\right) \cdot K_{i \lambda}(\rho r) \cdot \frac{2 \sinh \left[\lambda\left(\alpha-\theta^{\prime}\right)\right]}{\cosh (\lambda \alpha)} d \lambda .
$$

For short-period pulses we use the classical approximation (Erdelyi, et al., 1953).

$$
\begin{aligned}
I_{-i \lambda}\left(\rho r^{\prime}\right) & =\frac{e^{\xi^{\prime}}}{\sqrt{2 \pi}\left[\left(\rho r^{\prime}\right)^{2}-\lambda^{2}\right]^{1 / 4}}\left[1+0\left(\lambda^{-1}\right)\right] \\
K_{i \lambda}(\rho r) & =\frac{e^{-\xi}}{\sqrt{2 / \pi}\left[(\rho r)^{2}-\lambda^{2}\right]^{1 / 4}}\left[1+0\left(\lambda^{-1}\right)\right]
\end{aligned}
$$

where

$$
\begin{aligned}
& \xi^{\prime} \equiv\left[\left(\rho r^{\prime}\right)^{2}-\lambda^{2}\right]^{1 / 2}+\lambda \sin ^{-1}\left(\lambda / \rho r^{\prime}\right) \\
& \xi \equiv\left[(\rho r)^{2}-\lambda^{2}\right]^{1 / 2}+\lambda \sin ^{-1}(\lambda / \rho r)
\end{aligned}
$$

Thus we get

$$
\begin{aligned}
\bar{W} \cong-i \int_{-\infty}^{\infty} \frac{e^{\xi^{\prime}-\xi}}{\left[\left(\rho r^{\prime}\right)^{2}-\lambda^{2}\right]^{1 / 4}\left[(\rho r)^{2}-\lambda^{2}\right]^{1 / 4}} \cdot \frac{\sinh \left[\lambda\left(\alpha-\theta^{\prime}\right)\right]}{\cosh \lambda \alpha} d \lambda \\
\cong-i \int_{-\infty}^{\infty} \frac{e^{\xi^{\prime}-\xi}}{\left[\left(\rho r^{\prime}\right)^{2}-\lambda^{2}\right]^{1 / 4}\left[(\rho r)^{2}-\lambda^{2}\right]^{1 / 4}} \\
\quad\left\{\sum_{n=0}^{N_{1}}(-1)^{n} \exp \left(-\lambda \theta^{\prime}-2 n \alpha \lambda\right)+\sum_{n=1}^{N_{2}}(-1)^{n} \exp \left(\lambda \theta^{\prime}-2 n \alpha \lambda\right)\right. \\
+\frac{(-1)^{N_{1}+1} \cosh \left[\lambda \pi-\lambda \theta^{\prime}-\left(2 N_{1}+1\right) \alpha \lambda\right]}{2 \sinh \lambda \pi \cosh \lambda \alpha} \\
\left.\quad+\frac{(-1)^{N_{2}+1} \cosh \left[\lambda \pi+\lambda \theta^{\prime}-\left(2 N_{2}+1\right) \alpha \lambda\right]}{2 \sinh \lambda \pi \cosh \lambda \alpha}\right\} d \lambda
\end{aligned}
$$

where $N_{1}, N_{2}$ are the largest positive integers to keep $\theta^{\prime}+2 N_{1} \alpha<\pi$ and $-\theta^{\prime}+$ $2 N_{2} \alpha<\pi$.

Now, the only non-analytical regions are the branch cuts along the real axis; we distort the integral path to the imaginary axis and by using the principle of Schwarz reflection we obtain

$$
\begin{aligned}
\bar{W} \cong 2 \operatorname{Im}\left\{\sum_{n=0}^{N_{1}} \int_{0}^{i \infty}(-1)^{n} \frac{\exp \left[-s\left(\phi+\theta^{\prime} \gamma+2 n \alpha \gamma\right)\right]}{\left[\left(r^{\prime} / \beta\right)^{2}-\gamma^{2}\right]^{1 / 4}\left[(r / \beta)^{2}-\gamma^{2}\right]^{1 / 4}} d \gamma\right. \\
+\sum_{n=1}^{N_{2}} \int_{0}^{i \infty}(-1)^{n} \cdot \frac{\exp \left[-s\left(\phi-\theta^{\prime} \gamma+2 n \alpha \gamma\right)\right]}{\left[\left(r^{\prime} / \beta\right)^{2}-\gamma^{2}\right]^{1 / 4}\left[(r / \beta)^{2}-\gamma^{2}\right]^{1 / 4}} d \gamma \\
+\int_{0}^{i \infty} \frac{(-1)^{N_{1}+1} e^{-s \phi} \cosh \left[s \gamma \pi-s \gamma \theta^{\prime}-s \gamma\left(2 N_{1}+1\right) \alpha\right]}{2 \sinh (s \gamma \pi) \cosh (s \gamma \alpha)\left[\left(r^{\prime} / \beta\right)^{2}-\gamma^{2}\right]^{1 / 4}\left[(r / \beta)^{2}-\gamma^{2}\right]^{1 / 4}} d \gamma \\
\left.+\int_{0}^{i \infty} \frac{(-1)^{N_{2}+1} e^{-s \phi} \cosh \left[s \gamma \pi+s \gamma \theta^{\prime}-s \gamma\left(2 N_{2}+1\right) \alpha\right]}{2 \sinh (s \gamma \pi) \cosh (s \gamma \alpha)\left[\left(r^{\prime} / \beta\right)^{2}-\gamma^{2}\right]^{1 / 4}\left[(r / \beta)^{2}-\gamma^{2}\right]^{1 / 4}}\right\} d \gamma
\end{aligned}
$$


where $\gamma \equiv \lambda / s$

$$
\phi \equiv\left[(r / \beta)^{2}-\gamma^{2}\right]^{1 / 2}-\left[\left(r^{\prime} / \beta\right)^{2}-\gamma^{2}\right]^{1 / 2}+\gamma \sin ^{-1}(\gamma \beta / r)-\gamma \sin ^{-1}\left(\gamma \beta / r^{\prime}\right) .
$$

The first two terms are the summations of multiples trapped between the two boundaries. The other two terms are the diffracted waves from the wedge tip, which we will neglect for reasons which will become apparent later.

Then by Cagniard-de Hoop method we have

$$
W \cong \sum_{n=1}^{N} W_{n}=\sum_{n=1}^{N} \operatorname{Im}\left\{\frac{2 \Re n}{\left[\left(r^{\prime} / \beta\right)^{2}-\gamma^{2}\right]^{1 / 4}\left[(r / \beta)^{2}-\gamma^{2}\right]^{1 / 4}} \frac{d \gamma}{d t}\right\}_{\Gamma_{n}}
$$

where $\Gamma_{n}$ is the contour in the complex $\gamma$-plane defined by

$$
\begin{cases}\phi+\theta^{\prime} \gamma+(n-1) \alpha \gamma=\text { pure real, } & \text { if } n=\text { odd } \\ \phi-\theta^{\prime} \gamma+(n) \alpha \gamma=\text { pure real, } & \text { if } n=\text { even }\end{cases}
$$

and,

$$
\begin{aligned}
& N=N_{1}+N_{2}+1 \\
& \mathcal{R} n=(-1)^{n-1}
\end{aligned}
$$

The factor ( $2 \Omega n$ ) is the reflection effect, $R n$ is the product of all reflection coefficients which are either 1 at the free surface or $(-1)$ at the rigid boundary. The factor 2 is the free surface effect, and the factor

$$
\frac{d \gamma / d t}{\left[\left(r^{\prime} / \beta\right)^{2}-\gamma^{2}\right]^{1 / 4}\left[(r / \beta)^{2}-\gamma^{2}\right]^{1 / 4}}
$$

controls the spreading effect.

Next, we illustrate the behavior of these generalized rays by examining the firstmotion approximation of a particular ray diagrammed in Figure 2 with

$$
\begin{aligned}
t(\gamma)= & {\left[(r / \beta)^{2}-\gamma^{2}\right]^{1 / 2}-\left[\left(r^{\prime} / \beta\right)^{2}-\gamma^{2}\right]^{1 / 2}+\gamma \sin ^{-1}(\gamma \beta / r)-\gamma \sin ^{-1}\left(\gamma \beta / r^{\prime}\right) } \\
& +\left(2 \alpha-\theta^{\prime}\right) \gamma .
\end{aligned}
$$

The geometric time, $t_{0}$, is obtained by setting $\partial t / \partial \gamma=0$ and solving for appropriate $\gamma_{0}$,

$$
d t / d \gamma=\left(2 \alpha-\theta^{\prime}\right)+\sin ^{-1} \gamma \beta / \gamma-\sin ^{-1} \gamma \beta / r^{\prime}
$$

But since $\epsilon^{\prime}-\epsilon=2 \alpha-\theta^{\prime}$ one obtains

$$
\gamma_{0}=\left(r^{\prime} \sin \epsilon^{\prime} / \beta\right)=(r \sin \epsilon / \beta) .
$$

Substituting $\gamma_{0}$ into (13) one obtains

$$
t_{0}=(\overline{A B}+\overline{B C}) / \beta
$$


Following the usual first-motion technique, one approximates

$$
\frac{d \gamma}{d t} \cong \frac{i\left(t-t_{0}\right)^{-1 / 2}}{\left(2\left|\frac{\partial^{2} t}{\partial \gamma^{2}}\right|\right)^{1 / 2}}
$$

which can be written

$$
\frac{d \gamma}{d t}=\frac{i \sqrt{\beta}\left[\left(r^{\prime} / \beta\right)^{2}-\gamma^{2}\right]^{1 / 4}\left[(r / \beta)^{2}-\gamma^{2}\right]^{1 / 4}}{\left[2\left(t-t_{0}\right)(\overline{A B}+\overline{B C})\right]^{1 / 2}}
$$

and evaluating $W_{n}$ from (11) we obtain

$$
W_{n} \alpha\left(t^{2}-t_{0}^{2}\right)^{-1 / 2} H\left(t-t_{0}\right)
$$

which is the behavior of the well-known line-source solution, see Gilbert and Knopoff (1961).

Thus, we obtain a solution consisting of generalized rays similar to the flat case except that the number of rays is finite, limited by conditions on $N_{1}$ and $\mathrm{N}_{2}$-see

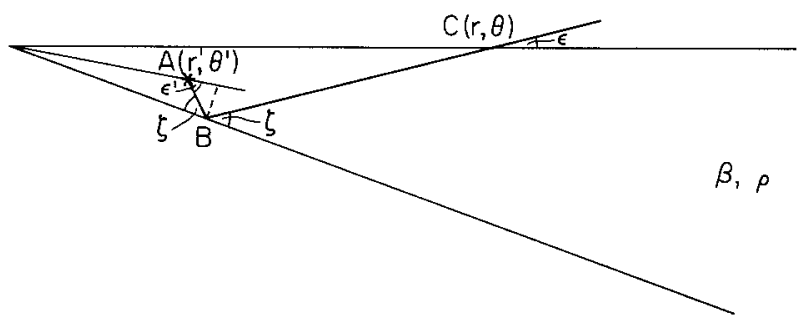

FIg. 2. Diagram showing the geometric path of a particular ray reflecting off the lower boundary following path $\mathrm{ABC}$.

appendix for details. There are also two remainder terms given in expression (10) that correspond to diffracted waves which do not have saddle points and are depleted in the shorter periods as discussed by Hudson (1963). When the dip is small the number of contributing rays approaches that normally used in the flat case. However, when the dip is large the number of rays allowed is relatively small and the remainder terms are proportionately larger.

Method of equivalent models. If we drop the term (2Rn) in formula (11), we can find an equivalent model for each generalized ray. For example, suppose we examine the ray with its geometric path as shown in Figure 3. It is easy to show that the path in the layer is equivalent to the ray path with $B_{0} B_{4}{ }^{\prime}$ in a whole-space model. Furthermore, it is convenient to choose the coordinate system $(x, y, z)$ and to use the ray parameter, $p$, defined by

$$
t(p)=p d+\left[\left(1 / \beta^{2}\right)-p^{2}\right]^{1 / 2} h=\text { pure real }
$$

At the geometric arriving time, $t_{0}, p$ becomes $\sin (\theta) / \beta$. For a $p$ along the de Hoop contour we assign a complex angle to it such that $p=\sin (a+b i) / \beta$ and the local 
ray parameters are defined as

$$
p_{m}=\frac{\sin [a+(m-1) \alpha+b i]}{\beta}
$$

Thus, the $S H$ displacement of the $n$th generalized ray becomes

$$
W_{n}=\left.\operatorname{Im}\left\{\frac{2 \mathfrak{R} d p / d t}{\left[\left(1 / \beta^{2}\right)-p^{2}\right]^{1 / 2}}\right\}\right|_{p \text { along } \Psi_{n}}
$$

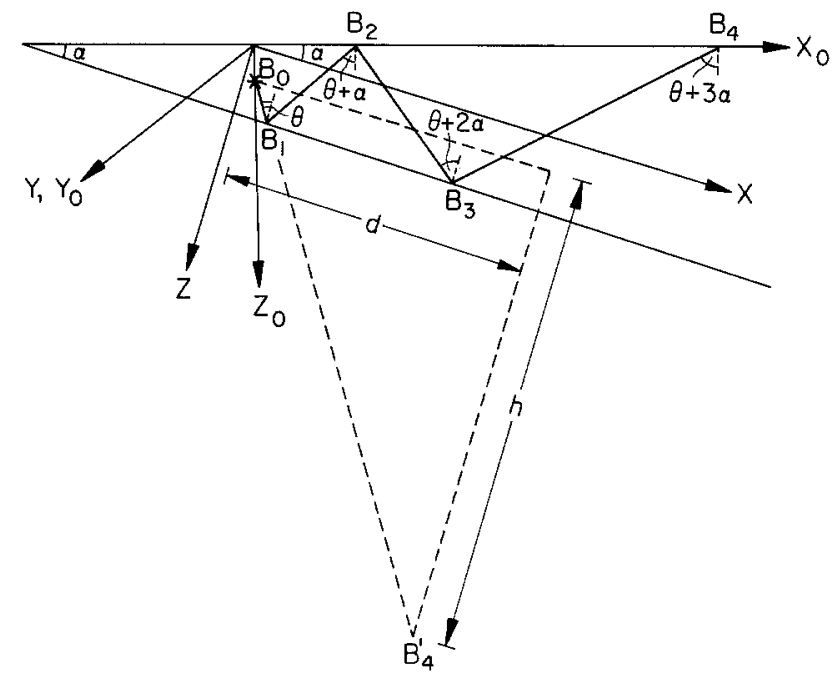

FIg. 3. Diagram showing an equivalent model of a ray where the length of the line segment between points $B_{0}$ and $B_{4}{ }^{\prime}$ is taken to be the sum of the segments $B_{0} B_{1}, B_{1} B_{2}, B_{2} B_{3}$, and $B_{3} B_{4}$.

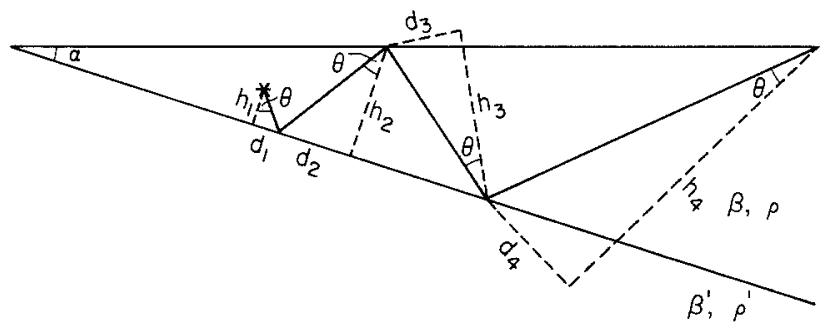

FIG. 4. Diagram showing one kind of parameterization for a ray.

where $R$ is the product of all the reflection coefficients in terms of local ray parameters $P_{m}$ 's and $\Psi_{n}$ is the appropriate de-Hoop contour for each ray in its equivalent model.

Following another point of view we can break the parameters $d$ and $h$ into smaller segments as shown in Figure 4. This gives

$$
d=d_{1}+d_{2}+d_{3}+d_{4}
$$

and

$$
h=h_{1}+h_{2}+h_{s}+h_{4}
$$

Now we can write the de-Hoop contour (20) in the following way

$$
\sum_{m=1}^{4}\left(p d_{m}+\left[\left(1 / \beta^{2}-p^{2}\right)\right]^{1 / 2} h_{m}\right)=\text { pure real }
$$


We note that under an arbitrary rotation of local frames, which changes the real parts of the complex angles, the quantity $t_{m}=d_{m} \sin \left(a_{m}+b_{m} i\right)+h_{m} \cos \left(a_{m}+b_{m} i\right)$ is invariant, where $d_{m}$ and $h_{m}$ are defined as the horizontal and vertical projections of the segment of the geometric ray path in a frame, see Figure 5 . Note that

$$
\begin{aligned}
& d \sin (a)+h \cos (a)=d^{\prime} \sin \left(a^{\prime}\right)+h^{\prime} \cos \left(a^{\prime}\right) \\
& d \cos (a)-h \sin (a)=d^{\prime} \cos \left(a^{\prime}\right)-h^{\prime} \sin \left(a^{\prime}\right)
\end{aligned}
$$

and thus,

$$
d \sin (a+b i)+h \cos (a+b i)=d^{\prime} \sin \left(a^{\prime}+b i\right)+h^{\prime} \cos \left(a^{\prime}+b i\right)
$$

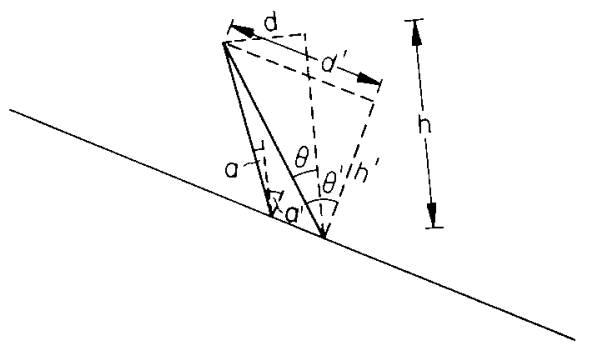

FIG. 5. Diagram illustrates formulas (25) and (26).

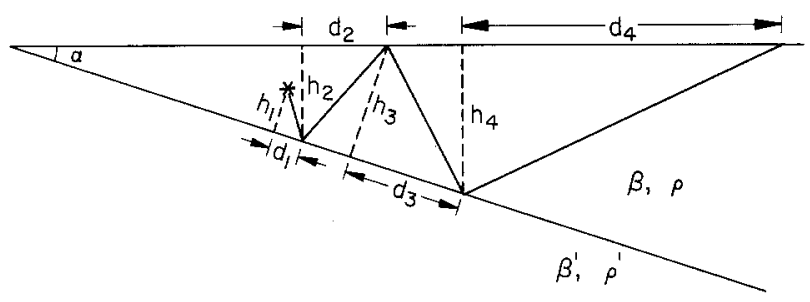

Fig. 6. Diagram showing another kind of parameterization for a ray.

Therefore, we can reconstruct the contour (24) by redefining $d_{m}$ and $h_{m}$ in the following way shown in Figure 6.

$$
\sum_{m=1}^{4}\left(d_{m} p_{m}+\left[(1 / \beta)^{2}-p_{m}{ }^{2}\right]^{1 / 2} h_{m}\right)=\text { pure real }
$$

where $p_{m}$ is the local ray parameters defined in (21). The $S H$ displacement of the $n$th generalized ray becomes

$$
W_{n}=\left.\operatorname{Im}\left\{\frac{2 \Omega\left(d p_{1} / d t\right)}{\left[(1 / \beta)^{2}-p_{1}^{2}\right]^{1 / 2}}\right\}\right|_{p_{1} \text { along } \Psi_{n}}
$$

where $\Psi_{n}$ is the de-Hoop contour, i.e., equation (28), of the $n$th generalized ray.

When the source is in the half-space, we can similarly build up the de-Hoop contour as shown in Figure 7.

$$
\sum_{m=1}^{4}\left(d_{m} p_{m}+\left[\left(1 / \beta^{2}\right)-p_{m}{ }^{2}\right]^{1 / 2} h_{m}\right)=\text { pure real, }
$$


$p_{m}$ 's are related to each other in the following way

$$
\begin{aligned}
& p_{1}=\sin \left(a^{\prime}+b^{\prime} i\right) / \beta^{\prime}=\sin (a+b i) / \beta \\
& p_{m}=\sin (a+(m-1) \alpha+b i) / \beta \quad \text { for } m=2,3,4
\end{aligned}
$$

The $S H$-displacement of the ( $n$ th) generalized ray is

$$
W_{n}=\left.\operatorname{Im}\left\{\frac{2 J \Re\left(d p_{1} / d t\right)}{\left[(1 / \beta)^{2}-p_{1}^{2}\right]^{1 / 2}}\right\}\right|_{p_{1} \text { along } \Psi_{n}}
$$

where $\mathcal{T}$ is the transmission coefficient in terms of local ray parameters.

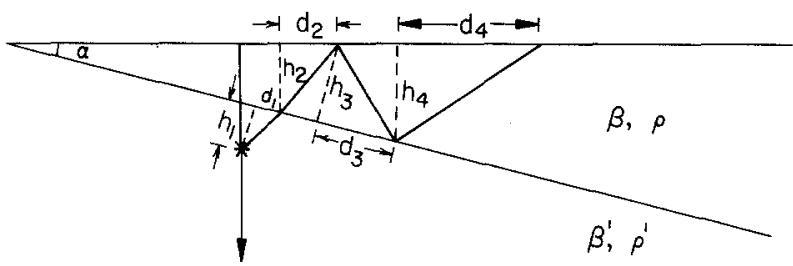

7. FIG. Diagram showing the parameterization of a ray with the source in the half-space.

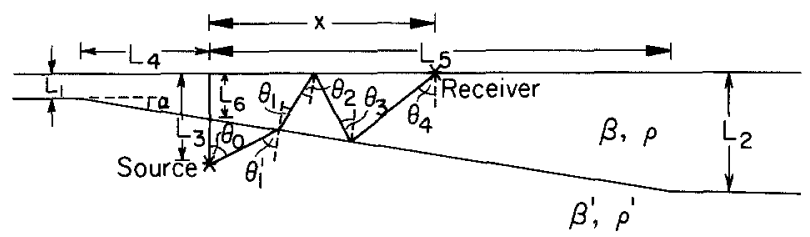

FIG. 8. Diagram showing the labeling of model parameters used in Figure 8 showing synthesis of seismograms.

It should be noted that when we relax the rigid bottom allowing an elastic boundary, we replaced on $n$ by the product of elastic reflection-transmission coefficients in terms of the complex local ray parameters. This approximation is essentially extended from the geometric ray theory in general structures. This enables us to evaluate nongeometric arrivals which play important roles around geometric times of multiples with critical reflections. Since this approximation lacks a rigorous theoretical basis, we need some independent checks. A comparison of results against a finite element code has been done and found to be generally accurate as discussed by Hong and Kosloff (1977).

\section{Numerical Seismograms}

In this section we present some numerical results of $S H$-wave propagation in a layer over a half-space. The model is given in Figure 8 where we have attempted to minimize diffraction effects by removing the wedge tip. We assumed a simple sawtooth time function with a 3 -sec duration. The results are displayed in Figures 9 through 12 , in which the columns on the left and middle contain individual contributions of generalized rays 1 through 4 with the summation given on the right. The higher-order multiples were not included here since they are small and arrive somewhat later. All the traces on each figure are on the same amplitude scale to properly show the ray contributions.

In Figure 9, we fixed the source and receiver at $x=20 \mathrm{~km}$ and changed the dipping 
angle from $0^{\circ}$ to $10^{\circ}$ where the layer thickens toward the receiver, shooting down-dip. The motion behaves as if the source was actually in the layer for the larger dip angles. This effect is achieved by the simple fact that each time a ray bounces in the layer its trajectory becomes flatter and can easily reach critical angle, thus producing head waves. The separation between the direct ray and multiples is increased compared to the flat case since the layer thickens toward the receiver. In this way the summation

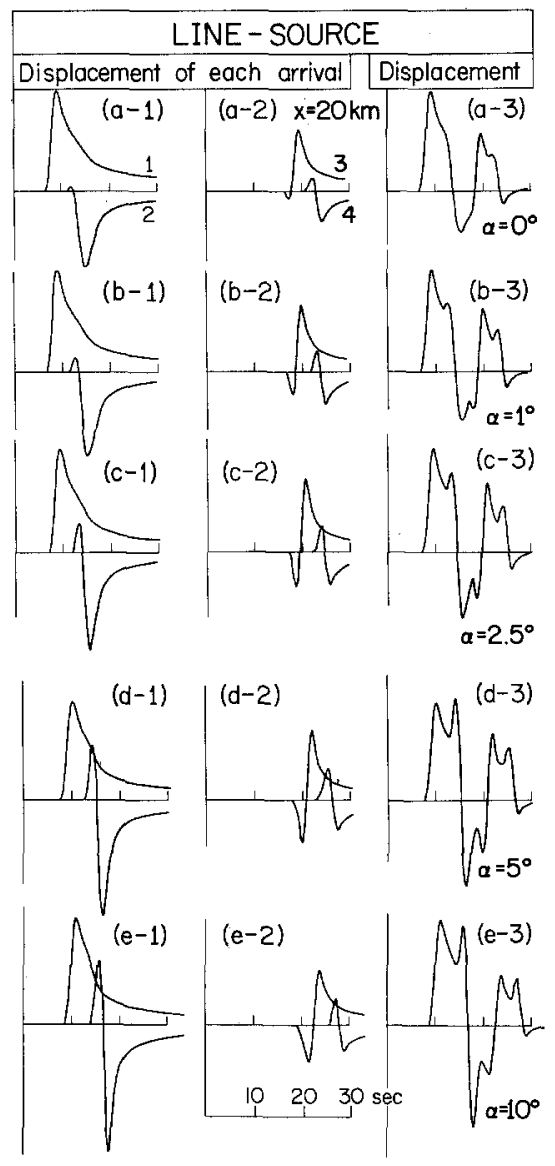

Fra. 9. Theoretical displacements for the down-dip case with $\alpha=0^{\circ}$ (a-3), $\alpha=1^{\circ}(\mathrm{b}-3), \alpha=$ $2.5^{\circ}$ (c-3), etc. Individual ray contributions are labeled $1,2,3$, and 4 indicating the direct, (1), followed by the one multiple, (2), etc. Column (a-1) contains rays (1) and (2) and column (a-2) contains rays (3) and (4). The summation of these four rays is given in (a-3) and similarly for the other dip angles. The common model parameters are $L_{3}=6 \mathrm{~km}, L_{4}=10 \mathrm{~km}, L_{5}=50 \mathrm{~km}$, $L_{6}=4 \mathrm{~km}, x=20 \mathrm{~km}, \beta=1.6 \mathrm{~km} / \mathrm{sec}, \beta^{\prime}=4.8 \mathrm{~km} / \mathrm{sec}, \rho=2.6 \mathrm{gm} / \mathrm{cm}^{3}, \rho^{\prime}=2.7 \mathrm{gm} / \mathrm{cm}^{3}$. The other parameters for the individual cases are: $L_{1}=4,3.825,3.563,3.125,2.242 \mathrm{~km}$ and $L_{2}=4$, $4.873,6.183,8.376,12.789 \mathrm{~km}$ for $\mathrm{a}, \mathrm{b}, \mathrm{c}, \mathrm{d}$, and e, respectively.

of rays produces dispersion and the development of Love waves. The opposite situation occurs when shooting up-dip, layer thinning toward the receiver. This case is displayed in Figure 10 where we again fix the source and receiver at $x=20 \mathrm{~km}$ and vary the dip angle from $0^{\circ}$ to $-5^{\circ}$. In this situation the motion behaves as if the source were somewhat deeper than it actually is when compared to the flat layer result. This effect is produced because each time a ray bounces in the layer its trajectory becomes steeper and radiates energy into the half-space and becomes ineffectual.

One of the more interesting features produced by the dipping layer is back-scattered 
rays. When shooting up-dip, rays can either enter the layer beyond the receiver and, after bouncing a few times, become steeper and turn back toward the receiver. But, in general, these rays are not very large contributors because of the large loss of energy in entering the layer at these large angles of incidence.

When shooting down-dip, rays can leave the source moving away from the receiver and, after bouncing a few times in the layer, reverse their direction and return to the receiver. An example of this type of ray is given in Figure 11 when the dip-angle is set at $\alpha=10^{\circ}$ and $x$ is increased from 10 to $30 \mathrm{~km}$. We have included some critical values of $\theta_{1}$ and $\theta_{1}^{\prime}$ for ray 4 where these angles are negative. These back-scattered rays are not particularly impressive here, but they could become important in the

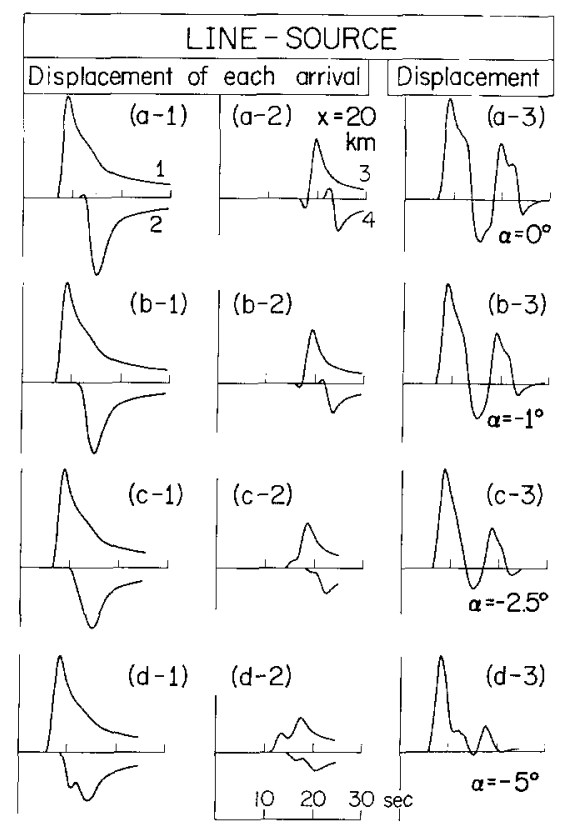

FIG. 10. Theoretical displacements for the up-dip case with $\alpha=0^{\circ},-1^{\circ},-2.5^{\circ},-5^{\circ}$, respectively. The common model parameters are: $L_{3}=6 \mathrm{~km}, L_{4}=10 \mathrm{~km}, L_{5}=23 \mathrm{~km}, L_{6}=4 \mathrm{~km}$, $x=20 \mathrm{~km}, \beta=1.6 \mathrm{~km} / \mathrm{sec}, \beta^{\prime}=4.8 \mathrm{~km} / \mathrm{sec}, \rho=2.6 \mathrm{gm} / \mathrm{cm}^{3}, \rho^{\prime}=2.7 \mathrm{gm} / \mathrm{cm}^{3}$. The other parameters for the individual cases are: $L_{1}=4,4.175,4.437,4.875 \mathrm{~km} ; L_{2}=4,3.599,2.996,1.989 \mathrm{~km}$, for $a, b, c$, and $d$, respectively.

presence of radiation pattern. A profile similar to Figure 11 but shooting up-dip is given in Figure 12 for completeness.

An interesting feature displayed by some of these rays is a tunneling effect caused by a sharp bend in the de-Hoop contour where the ray parameter remains almost real between the geometric time and the corresponding time for a source on the boundary. It is the typical behavior of a ray incident from fast region to slow region at a large angle-see Helmberger and Malone (1975). In our present case of shooting up-dip, the rays are more grazing than the corresponding ones in the down-dip case. This is the reason why the tunneling effect becomes important in the up-dip situation; for example, notice the apparent double arrival of ray 2 in Figure $12(c-1)$ and $(d-1)$.

\section{Discussion}

At this point, we will review the approximations made in above techniques followed by a comparison of our results with those obtained from the finite element method. 
First, we neglected the diffracted waves associated with the wedge tip by removing the tip from the model (see Figure 8). Second, we used a high-frequency approximation given by expression (8), and third, we replaced the rigid boundary by an elastic one. Errors associated with the first and third are difficult to determine analytically, and, therefore, a series of numerical comparisons were performed to test the overall va-

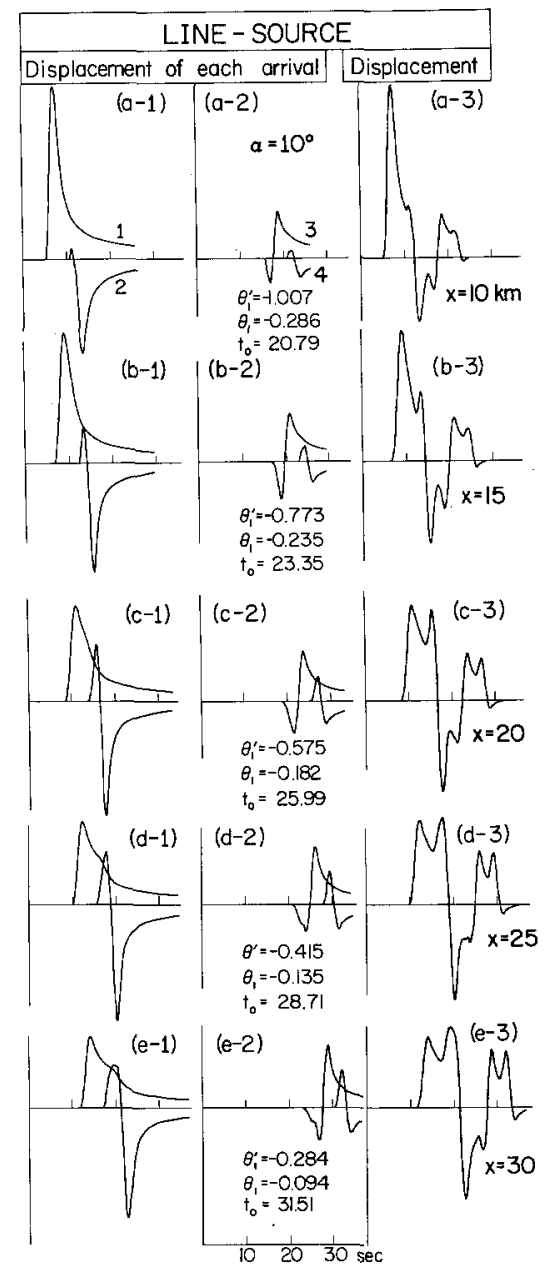

FIG. 11. The common model parameters are $L_{1}=2.242 \mathrm{~km}, L_{2}=12.789 \mathrm{~km}, L_{3}=6 \mathrm{~km}, L_{4}=$ $10 \mathrm{~km}, L_{5}=50 \mathrm{~km}, L_{6}=4 \mathrm{~km}$, and the other parameters for individual cases are: $x=10,15,20$, $25,30 \mathrm{~km}$, for $\mathrm{a}, \mathrm{b}, \mathrm{c}, \mathrm{d}$, and e, respectively.

lidity-see Hong and Kosloff (1977). An example of such a comparison is given in Figure 13 where the wave form of Figure (11b) is compared with a first-motion approximation as well. The comparison is somewhat difficult because of the limitations associated with each method, in that the finite element technique loses accuracy at the shorter periods caused by the element size whereas the GRT technique is not accurate at the longer periods. We attempted to remedy the situation by comparing the results after a convolution with a broad-band instrument which eliminated the extreme periods-see Figure 3 of Helmberger and Malone (1975) for a description of the instrument response. The convoluted wave forms are shown in Figure 13 under the title of synthetic. The correspondence is sufficiently good to show the usefulness 


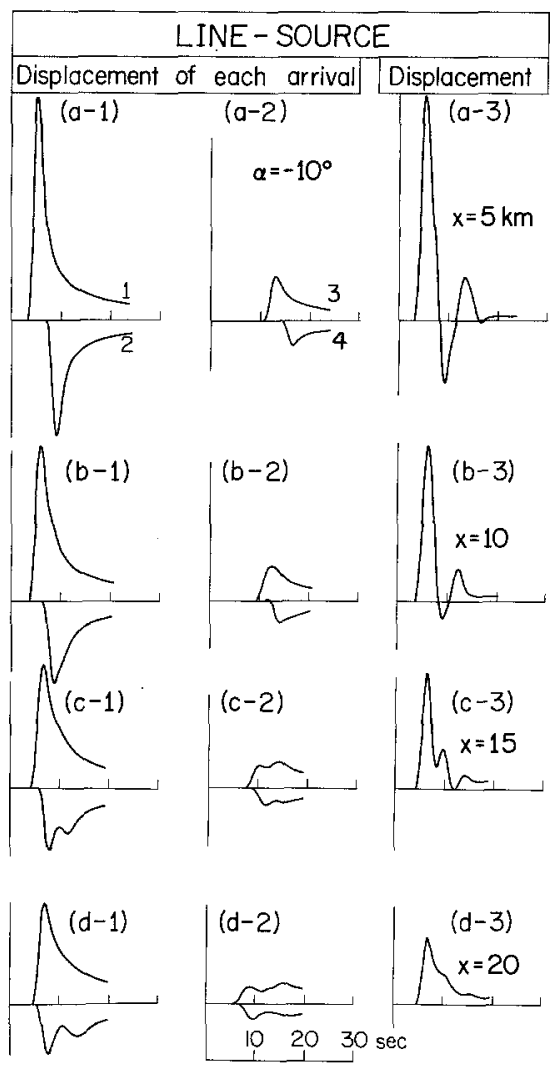

FIG. 12. The common model parameters are $L_{1}=5.758 \mathrm{~km}, L_{2}=0.484 \mathrm{~km}, L_{3}=6 \mathrm{~km}, L_{4}=$ $10 \mathrm{~km}, L_{5}=22 \mathrm{~km}$ and the other parameters for individual cases are: $x=5,10,15,20 \mathrm{~km}$ for a, $b, c$, and $d$, respectively.

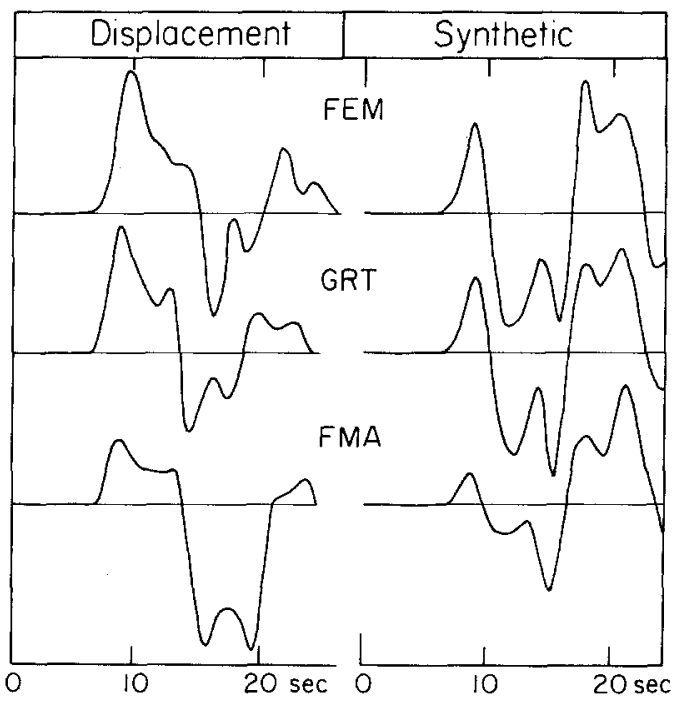

FIG. 13. Comparison of displacements and corresponding synthetics for identical models by three different methods : finite element method, FEM, the top view; generalized ray theory, GRT, the middle row; and first-motion approximation, FMA, the bottom view. 
of our technique but more detailed tests will be presented later. The comparison with geometric ray theory, essentially the same as the first-motion approximations of GRT, shows the effects of the rapid change of the transmission coefficient with frequency associated with the direct arrival. This feature is related to the ratio of the source duration to travel time and becomes small as the ratio goes to zero. Thus, the results presented by Langston (1977) are in agreement with GRT as discussed in this paper in the limit of constant ray parameter or large travel times.

\section{ConcLusion}

A detailed investigation of the wedge problem has confirmed earlier studies in that the motion can be constructed from rays when the diffracted wave from the wedge tip can be neglected. But instead of stopping with geometric ray theory as in the classical treatments, we have developed a scheme of mapping the de-Hoop contour by using the local complex ray parameters, thus allowing the application of generalized ray theory. This result allows us to obtain an accurate picture of the motions over a wide frequency band. Numerical motions are presented for a layer over a half-space which display some of the important features of dipping structure as revealed by their accompanying ray expansions. The results indicate that shooting down-dip allows rapid development of Love waves by the trapping of energy in the layer. The opposite result occurs when shooting up-dip. We think there are abundant examples of this type of phenomenon observed in local earthquake records and that this technique provides a new dimension in solving these seismological problems.

\section{ACKNOWLEDGMENTS}

The authors thank D. Kosloff for providing us the results of finite element method for comparison. They also thank H. Kanamori, D. Harkrider, and C. Langston for reading the manuscript and giving constructive suggestions. This research was supported in part by the National Science Foundation under Grant ENV76-10506 and in part by the Advanced Projects Research Agency at the Department of Defense and was monitored by the Air Force Office of Scientific Research under Contract F44620-72-C-0078.

\section{REFERENCES}

Erdelyi, A., W. Magnus, F. Oberhettinger, and F. G. Tricomi (1953). Higher Transcendental Functions, Vol. 2, Bateman Manuscript Project, McGraw-Hill, New York.

Gilbert, F. and L. Knopoff (1961). The directivity problem for a buried line source, Geophysics 26, 626-634.

Helmberger, D. V. and S. D. Malone (1975). Modeling local earthquakes as shear dislocations in a layered halfspace, J. Geophys. Res. 80, 4881-4888.

Hudson, J. A. (1963). SH waves in a wedged-shaped medium, Geophys. J . 7, 517-546.

Hong, T. L. and D. Kosloff (in preparation). Numerical seismograms in dipping structure, a comparison of generalized ray theory and finite element method.

Langston, C. A. (1977). The effect of planar dipping structure on source and receiver responses for constant ray parameter, Bull. Seism. Soc. Am. 67, 1029-1050.

Oberhettinger, F. (1954). Diffraction of waves by a wedge, Commun. Pure and Appl. Math. 7, $551-563$.

Setsmological LaboRatory

Division of Geological and Planetary Sciences

California Institute of Technology

Pasadena, California 91125

Contribution No. 2836

Manuscript received January 26, 1977 


\section{APPENDIX}

Some of the details in the derivation of formula (9) were skipped in the main context. Since we think some readers might be interested in how the limitation $N_{1}$ and $N_{2}$ came about, we add this appendix.

$$
\begin{aligned}
& \frac{\sinh \left[\lambda\left(\alpha-\theta^{\prime}\right)\right]}{\cosh (\lambda \alpha)}=\frac{2 \sinh (\lambda \pi) \sinh \left[\lambda\left(\alpha-\theta^{\prime}\right)\right]}{2 \sinh (\lambda \pi) \cosh (\lambda \alpha)} \\
& =\frac{\cosh \left[\lambda \pi+\lambda \alpha-\lambda \theta^{\prime}\right]-\cosh \left[\lambda \pi-\lambda \alpha+\lambda \theta^{\prime}\right]}{2 \sinh (\lambda \pi) \cosh (\lambda \alpha)} \\
& =\sum_{n=0}^{N_{1}} \frac{(-1)^{n} \cosh \left[\lambda \pi-\lambda \theta^{\prime}-2 n \alpha \lambda\right]}{\sinh (\lambda \pi)} \\
& +\frac{(-1)^{N_{1}+1} \cosh \left[\lambda \pi-\lambda \theta^{\prime}-\left(2 N_{1}+1\right) \alpha \lambda\right]}{2 \sinh (\lambda \pi) \cosh (\lambda \alpha)} \\
& -\sum_{n=0}^{N_{2}} \frac{(-1)^{n-1} \cosh \left[\lambda \pi+\lambda \theta^{\prime}-2 n \alpha \lambda\right]}{\sinh (\lambda \pi)} \\
& +\frac{(-1)^{N_{2}} \cosh \left[\lambda \pi+\lambda \theta^{\prime}-\left(2 N_{2}+1\right) \alpha \lambda\right] .}{2 \sinh (\lambda \pi) \cosh (\lambda \alpha)} \\
& \underset{\lambda \gg 1}{\cong} \sum_{n=0}^{N_{1}} \frac{(-1)^{n} \exp \left[\lambda \pi-\lambda \theta^{\prime}-2 n \alpha \lambda\right]}{e^{\lambda \pi}} \\
& +\frac{(-1)^{N_{1}+1} \cosh \left[\lambda \pi-\lambda \theta^{\prime}-\left(2 N_{1}+1\right) \alpha \lambda\right]}{2 \sinh (\lambda \pi) \cosh (\lambda \alpha)} \\
& +\sum_{n=1}^{N_{2}} \frac{(-1)^{n} \exp \left[\lambda \pi+\lambda \theta^{\prime}-2 n \alpha \lambda\right]}{e^{\lambda \pi}} \\
& +\frac{(-1)^{N_{2}+1} \cosh \left[\lambda \pi+\lambda \theta^{\prime}-\left(2 N_{2}+1\right) \alpha \lambda\right]}{2 \sinh (\lambda \pi) \cosh (\lambda \alpha)}
\end{aligned}
$$

Here, the two conditions

$$
\pi-\theta^{\prime}-2 N_{1} \alpha>0 \text { and } \pi+\theta^{\prime}-2 N_{2} \alpha>0
$$

are required. 